\title{
DIEUDONNÉ-SCHWARTZ THEOREM ON BOUNDED SETS IN INDUCTIVE LIMITS. II
}

\author{
J. KUCERA AND C. BOSCH
}

\begin{abstract}
The Dieudonné-Schwartz Theorem [1, Chapter 2, §12] has been stated for strict inductive limits. In [3] it has been extended to inductive limits. Here the result of [3] is generalized. Also, the case when each set bounded in ind lim $E_{n}$ is contained, but not necessarily bounded, in some $E_{n}$ is considered.
\end{abstract}

Let $E_{1} \subset E_{2} \subset \cdots$ be a sequence of locally convex spaces and $E=$ ind $\lim E_{n}$ their inductive limit (with respect to the identity maps id: $E_{n} \rightarrow E_{n+1}$ ). The Dieudonne-Schwartz theorem states that a set $B \subset E$ is bounded if and only if it is contained and bounded in some $E_{n}$, provided that

$(\mathrm{H}-1)$ each $E_{n}$ is closed in $E_{n+1}$, and

$(\mathrm{H}-2)$ the topology of each $E_{n}$ equals the topology induced in $E_{n}$ by $E_{n+1}$. It is convenient to introduce some further hypotheses:

(H-3) each $E_{n}$ is closed in $E$,

(H-4) each convex and closed set in $E_{n}$ is closed in $E_{n+1}$,

(H-7) for any $n \in N$ there is $p \in N$ such that $\bar{E}_{n}^{E} \subset E_{n+p}$, where $\bar{E}_{n}^{E}$ is the closure of $E_{n}$ in $E$,

(H-8) for any closed hyperplane $F$ in $E_{n},\left(E_{n} \backslash F\right) \cap \bar{F}^{E_{n+1}}=\varnothing$,

(DS) each set $B$ bounded in $E$ is contained in some $E_{n}$, and

(DST) each set $B$ bounded in $E$ is contained and bounded in some $E_{n}$.

The following implications: $\mathrm{H}-1 \& 2 \Rightarrow \mathrm{H}-3, \mathrm{H}-3 \Rightarrow \mathrm{DS}, \mathrm{H}-4 \Rightarrow \mathrm{DST}$, and $\mathrm{H}-4 \Rightarrow$ $\mathrm{H}-3$, are known, see [1, Chapter 2, §12; 2 and 3].

THEOREM $1 . \mathrm{H}-7 \Rightarrow \mathrm{DS}$. If $E$ is metrizable, the implication can be reversed.

Proof. Assume H-7 and existence of a set $B$ bounded in $E$ which is not contained in any $E_{n}$. Choose a sequence $1=n_{1} \leqslant n_{2} \leqslant n_{3} \leqslant \cdots$ such that $\bar{E}_{n_{k}}^{E} \subset E_{n_{k+1}}$ and $b_{k} \in B \backslash E_{n_{k}}, k \in N$.

Since $b_{1} \neq 0$, there exists convex 0 -nbhd $G_{1}$ in $E$ such that $b_{1} \notin G_{1}+G_{1}$. Put $V_{1}=G_{1} \cap E_{n_{1}}$ and $W_{1}=\bar{V}_{1}^{E}$. Then $W_{1} \subset\left(G_{1}+G_{1}\right) \cap E_{n_{2}}$ and $b_{1} \notin W_{1}, \frac{1}{2} b_{2} \notin W_{1}$. Hence there exists convex 0 -nbhd $G_{2}$ in $E$ such that $b_{1}, \frac{1}{2} b_{2} \notin W_{1}+G_{2}+G_{2}$. Put $V_{2}=G_{2} \cap E_{n_{2}}$ and $W_{2}=\bar{V}_{1}+V_{2}^{E}$. Again $W_{2} \subset\left(W_{1}+G_{2}+G_{2}\right) \cap E_{n_{3}}$ and $b_{1}$, $\frac{1}{2} b_{2}, \frac{1}{3} b_{3} \notin W_{2}$, etc. When the sequence $\left\{W_{k}\right\}$ is constructed, then $W=$ $\cup\left\{W_{k} ; k \in N\right\}$ is a 0 -nbhd in $E$ which does not absorb $B$.

Received by the editors January 20, 1982.

1980 Mathematics Subject Classification. Primary 46A05.

Key words and phrases. Locally convex spaces, (strict) inductive limit, bounded set. 
Let $\left\{G_{p}\right\}$ be a nested base for the topology of $E$. Assume $\bar{E}_{1}^{E}$ is not contained in any $E_{p}$. Take $x_{p} \in \bar{E}_{1}^{E} \backslash E_{p}$ and $a_{p}>0$ such that $a_{p} x_{p} \in G_{p}, p \in N$. Then $B=$ $\cup\left\{a_{p} x_{p}, p \in N\right\}$ is bounded in $E$ and not contained in any $E_{p}$.

LEMMA 1. H-8 $\Leftrightarrow$ each $g \in E_{n}^{\prime}$ has a continuous extension to $E_{n+1}$.

Proof. Assume H-8 and take $g \in E_{n}^{\prime}, f \neq 0$. Choose $x_{0} \in E_{n}, f\left(x_{0}\right) \neq 0$ and put $F=F^{-1}(0)$. Since, by H-8, $x_{0} \notin \bar{F}^{E_{n+1}}$ there exists $g \in E_{n+1}^{\prime}$ such that $g\left(x_{0}\right)=f\left(x_{0}\right)$ and $g(x)=0$ for $x \in \bar{F}^{E_{n+1}}$, that is $g^{-1}(0) \supset F$ and $g$ is the sought extension of $g$.

Let $F$ be a closed hyperplane in $E_{n}$. Take $f \in E_{n}^{\prime}$ such that $f^{-1}(0)=F$. If $f$ has an extension $g$ to $E_{n+1}$ then for $x \in E_{n} \backslash G, g(x)=f(x) \neq 0$, and $x \notin g^{-1}(0)=\bar{F}^{E_{n+1}}$.

LEMMA 2. DS \& H-8 $\Rightarrow$ each set $B \subset E_{n}$ which is bounded in $E$ is bounded in $E_{n}$.

Proof. Assume $B \subset E_{n}$, bounded in $E$, but not bounded in $E_{n}$. Then $B$ is not weakly bounded in $E_{n}$ and there is $f_{0} \in E_{n}^{\prime}$ (real dual) which is not bounded on $B$. For each $k \in N$, take $b_{k} \in B, f_{0}\left(b_{k}\right)>k$. By induction, choose $f_{p} \in E_{n+p}^{\prime}$ so that $f_{p}$ is an extension of $f_{p-1}, p \in N$. Then $\cup\left\{f_{p}^{-1}(-\infty, 1) ; p \in N\right\}$ is a 0 -nbhd in $E$ which does not absorb $B$.

From Theorem 1 and Lemmas 1 and 2 it follows that:

THEOREM 2. $\mathrm{H}-7 \& 8 \Rightarrow \mathrm{DS} \& \mathrm{H}-8 \Rightarrow$ DST.

Proposition. $\mathrm{H}-4 \Leftrightarrow \mathrm{H}-3 \& 8 \Leftrightarrow \mathrm{H}-1 \& 8$.

Proof. Evidently the if implications hold. To complete the cycle, assume H-1 \& 8. Take a set $A$ closed and convex in $E_{n}$. Without loss of generality, we may assume $0 \in A$. Denote by $g_{f}$ a continuous extension of $f \in E_{n}^{\prime}$ to $E_{n+1}$. There exists $M \subset E_{n}^{\prime}$ such that $A=\bigcap\left\{f^{-1}(-\infty, 1] ; f \in M\right\}=\bigcap\left\{g_{f}^{-1}(-\infty, 1] ; f \in M\right\} \cap E_{n} \supset \bar{A}^{E_{n+1}}$, since $E_{n}$ is closed in $E_{n+1}$.

We have a diagram:

\begin{tabular}{|c|c|c|c|c|c|c|}
\hline & & $\begin{array}{c}3 \& 8 \\
\mathbb{\Downarrow}\end{array}$ & $\Rightarrow$ & $\begin{array}{c}7 \& 8 \\
\mathbb{1}\end{array}$ & $\Rightarrow$ & $\begin{array}{c}\text { DS \& } 8 \\
\Downarrow\end{array}$ \\
\hline \multirow[t]{3}{*}{$1 \& 2$} & $\Rightarrow$ & 4 & $\Rightarrow$ & $7 \& 8$ & $\Rightarrow$ & DST \\
\hline & & $\Downarrow$ & & $\Downarrow$ & & $\Downarrow$ \\
\hline & & 3 & $\Rightarrow$ & 7 & $\Rightarrow$ & DS \\
\hline
\end{tabular}

The following examples will show that $\mathrm{H}-7$ \& 8 do not imply H-4 and DST \& H-8 do not imply H-7.

EXAMPLE 1. Take a Banach space $X$ and its proper subspace $Y$ (with the inherited topology). Put $E_{2 n-1}=X^{n} \times\{0\}^{N}, E_{2 n}=X^{n} \times Y \times\{0\}^{N}, n \in N$, all with the product topology. Then $E=\cup\left\{E_{n} ; n \in N\right\} \subset X^{N}$ has the topology inherited from $X^{N}$, as well as all $E_{n}$. Hence H-8 holds. Further $\overline{E_{2 n}} E=\overline{E_{2 n+1}} E=E_{2 n+1}$ and H-7 holds. On the other hand, H-3 \& 4 do not hold, since $E_{2 n} E_{2 n+1}=E_{2 n+1} \neq E_{2 n}$.

EXAMPLE 2. Let $\mathscr{D}[-n, n]=\left\{f \in C^{\infty}(R) ; \quad\right.$ supp $\left.f \subset[-n, n]\right\}$ and $\mathscr{D}=$ ind $\lim \mathscr{D}[-n, n]$. For this inductive limit DST holds by Dieudonné-Schwartz Theorem. Take $\varphi \in \mathscr{D}$, supp $\varphi=[-1,1], A=\{\varphi((p+1) x / p q) ; p, q \in N\}$, and put $E_{n}=\operatorname{sp}(A \cup \mathcal{D}[-n, n]), n \in N$, where sp stands for the span. We equip each $E_{n}$ 
with the topology inherited from $\mathscr{D}$ and H-8 holds. Since $\mathscr{Q}[-n, n] \subset E_{n}$, DST holds for the ind $\lim E_{n}$. On the other hand the closure of $E_{n}$ in $E$ contains functions $\varphi\left(\frac{1}{q} x\right), q \in N$, and since $\varphi\left(\frac{1}{q} x\right) \notin E_{s}, s=1,2, \ldots, q-1, \mathrm{H}-7$ does not hold.

EXAmple 3. Let $X, Y$ be the same as in Example 1. Put $E_{n}=X^{n} \times Y^{n}$. Then $E=X^{N} \cap \cup\left\{E_{n} ; n \in N\right\}$ with the topology inherited from $X^{N}$. If $B$ is the closed unit ball in $X$, then $B^{N} \cap E$ is bounded in $E$ but not contained in any $E_{n}$. Hence DS and H-3 \& 7 do not hold. Further $\bar{E}_{n} E_{n+1}=E_{n+1}$ and H-1 \& 4 do not hold, either. On the other hand, H-2 \& 8 hold since the topology of $E_{n}$ is inherited from $E_{n+1}$.

EXAMPLE 4. Put $W(x)=\sqrt{1+x^{2}}, x \in(-\infty, \infty)$, and $E_{n}=\left\{f \in L^{2}(R) ;\|f\|^{2}=\right.$ $\left.\int_{R}\left|W^{-n} f\right|^{2} d x<+\infty\right\}$. The norm $\|\cdot\|_{n}$ makes $E_{n}$ into a Hilbert space. Since the set (2) from Example 2 is dense in each $E_{n}$, we have $E_{n+p}=\overline{\mathcal{Q}} E_{n+p} \subset \bar{E}_{n}^{E_{n+p}} \subset \bar{E}_{n}^{E}$ and $\mathrm{H}-1,2,3,4,7$ do not hold. But, by Theorem 4 in [2], DST holds.

To show that H-8 does not hold, take $f_{k}=W^{n} \chi_{[-k, k]} \in E_{n}$ and put $B=\left\{f_{k}\right.$; $k \in N\}$. Then $\left\|f_{k}\right\|_{n}^{2}=2 k$ and $B \subset E_{n}$. Further $\left\|f_{k}\right\|_{n+1}^{2} \leqslant \pi$ and $B$ is bounded in $E_{n+1}$. If H-8 held $B$ would be bounded in $E_{n}$, by Lemma 2, which is not true.

\section{REFERENCES}

1. J. Horváth, Topological vector spaces and distributions, Vol. 1, Addison-Wesley, Reading, Mass., 1966.

2. J. Kucera and K. McKennon, Bounded sets in inductive limits, Proc. Amer. Math. Soc. 69 (1978), 62-64.

3. __ Dieudonné-Schwartz Theorem on bounded sets in inductive limits, Proc. Amer. Math. Soc. 78 (1980), 366-368.

Department of Mathematics, Washington State University, Pullman, Washington 99164 